\title{
Clinical and pathological characteristics of primary focal segmental
}

\section{glomerulosclerosis in adult}

\author{
Received: $30 / 8 / 2015$
}

Accepted: 5/6/2016

\begin{abstract}
Background and objective: Focal segmental glomerulosclerosis shares overlapping patterns of injury with segmental consolidation and obliteration of glomerular architecture by the accumulation of collagenous extracellular matrix or by increased cellularity or both. This study aimed to investigate the patterns of primary focal segmental glomerulosclerosis in adults in Erbil. The specific objectives of this study included determining the frequency of histological variant of Focal segmental glomerulosclerosis, defining the clinical characteristics of the primary type in general and recognizing the clinical and pathological characteristics for each variant separately.

Methods: A cross-sectional, clinico-pathologic study was conducted for 50 patients attending the Nephrology Department in Erbil Teaching Hospital between March and December 2013. This study included patients of $>16$ years old with biopsy-proven idiopathic focal segmental glomerulosclerosis.

Results: A total of 50 patients were enrolled into this study. The median age of patients was 33 years, ranged from 18 to $54,31(62 \%)$ males and $19(38 \%)$ females. The frequency of histopathological variants was $80 \%$ not otherwise specific focal segmental glomerulosclerosis, 14\% glomerular tip lesion, and 6\% cellular type. Nephrotic syndrome and hypertension were the main presenting features $(92 \%$ and $68 \%$, respectively). The mean percentage of sclerosed glomeruli was $40 \%$ and the mean interstitial fibrosis and tubular atrophy was $24 \%$.
\end{abstract}

Conclusion: A not otherwise specific variant is a common morphological lesion in many glomerular and non-glomerular diseases, and it is just like a junk drawer of multiple glomerular alterations with this common pattern of the lesion.

Keywords: Primary Focal Segmental Glomerulosclerosis; Nephrotic syndrome; Hypertension.

\section{Introduction}

Focal segmental glomerulosclerosis (FSGS) was first described in 1957 by Arnold Rich. ${ }^{1}$ However, it was not until the 1970s that FSGS emerged as a separate clinico-pathological entity. ${ }^{2}$ The term FSGS is applied to a pathologically diverse group of glomerular lesions with heterogeneous clinical manifestations. Focal segmental glomerulosclerosis shares overlapping patterns of injury with segmental consolidation and obliteration of glomerular architecture by accumulation of collagenous extracellular matrix or by increased cellularity or both. ${ }^{3-5}$ Focal segmental glomerulosclerosis can present with asymptomatic proteinuria or nephrotic syndrome with or without renal insufficiency. A diagnosis of FSGS is confirmed only by histopathology findings, but it can also be suggested clinically. ${ }^{6}$ There is a broad range of estimates for progression to renal failure, remission rates of the nephrotic syndrome, and response to therapy of patients with FSGS. ${ }^{5}$ Focal segmental glomerulosclerosis accounts for approximately $20 \%$ of cases of the nephrotic syndrome in children and $40 \%$ of

* Ministry of Health, Erbil, Iraq.

** Department of Internal Medicine, College of Medicine, Hawler Medical University, Erbil, Iraq. 
such cases in adults, with an estimated incidence of 7 per 1 million. $^{7}$ It is the most common primary glomerular disorder causing end-stage renal disease in the United States, with a prevalence of $4 \%{ }^{8}$ Typically, FSGS is observed in persons aged 18-45 years, although no age group is exempted from the disease. ${ }^{9}$ Depending on the causes, FSGS can be classified as following: ${ }^{10}$

1. Primary (idiopathic) FSGS: when no underlying cause is found; usually presents as nephrotic syndrome. ${ }^{11}$

2. Secondary FSGS, when an underlying cause is identified; it usually presents with kidney failure and proteinuria. ${ }^{11}$ This is actually a heterogeneous group including numerous causes such as drugs like heroin, $^{12}$ bisphosphanates, ${ }^{13-14}$ and all interferon therapy; ${ }^{15}$ viruses like Hepatitis $B$ and human immunodeficiency virus (HIV). ${ }^{16-17}$

The individual components of the name (FSGS) refer to the appearance of the kidney tissue on biopsy; focal: only some of the glomeruli are involved (as opposed to diffuse), segmental: only part of each glomerulus is involved (as opposed to global), glomerulosclerosis: refers to scarring of the glomerulus (a part of the nephron). ${ }^{18}$ Pathologically; on light microscopy, the lesion of FSGS initially affect only a few glomeruli and are characterized by sclerosis (collapse and solidification) limited to a portion of the glomerulus, either at hilum or at the tip of the tuft, or at both levels. ${ }^{10}$ On electron microscopy, there is diffuse effacement of the foot processes of podocytes and no or only scanty electron dense deposits in the mesangium. ${ }^{19}$ In the primary FSGS, the foot process effacement diffuse and generalized affecting even those glomeruli which are not affected by a sclerosing lesion. In the secondary FSGS, the foot process effacement is less noticeable, and usually, affects $<50 \%$ of the glomerular capillary surface area. ${ }^{20}$ In some cases, the FSGS lesion can already be seen at renal biopsy at the onset of proteinuria, while in other patients initial biopsy shows only minimal changes and a full histological picture of FSGS may be seen only in repeated biopsies. ${ }^{21}$ Recently, a working formulation for the morphological classification of primary FSGS, the "Columbia classification," was proposed by D'Agati et al, ${ }^{10}$ that recognized five histological variants based on light microscopic observations as following: Collapsing variant, glomerular tip variant, cellular variant, perihilar variant and not otherwise specific (NOS) variant. To the best of the researchers' knowledge, no study has yet evaluated the clinical characteristics of FSGS and the exact frequency of FSGS variant in adults depending on Columbia classification in our locality; as the histopathologic variants of FSGS that could be etiologically and pathogenetically distinct, and therefore have different prognoses and optimum therapies. For example, the tip lesion variant of FSG may have a more benign course compared to other structural variants of FSGS. ${ }^{22-23}$ Conversely, the collapsing variant of FSGS (collapsing FSGS) is known to have an aggressive course, often with decreased renal function at presentation and rapid progression to renal failure. ${ }^{24}$ Therefore, this study aimed to investigate the patterns (clinical and pathological) of primary FSGS in adults in Erbil. The specific objectives of this study included determining the frequency of histological variant of FSGS, defining the clinical characteristics of primary FSGS in general and recognizing the clinical and pathological characteristics for each FSGS variant separately.

\section{Ethical consideration:}

The research protocol was reviewed and approved by Ethical Committee of the center of Iraqi Board of Medical Specialization in Medicine Department. The anonymity of the participants was preserved. 


\section{Methods}

\section{Design, setting and time of the study:}

A cross-sectional, clinico-pathologic study was conducted for 50 patients attending the Nephrology Department in Erbil teaching hospital between March to December 2013.

Inclusion \& exclusion criteria:

This study included patients of $>16$ years old with biopsy-proven idiopathic FSGS.

All the renal biopsy specimens were examined in the histopathological laboratory of Shorsh General Hospital in Sulaymaniyah. Clinical data is prospectively gathered from the time of renal biopsy diagnosis. The diagnosis of primary FSGS was established when there was no ultrastructural evidence for another primary glomerular disease or a systemic disease associated with secondary segmental glomerular sclerosis and when review of the medical records of each patient revealed no evidence of systemic disease associated with glomerulopathy including diabetes mellitus, long-term hypertension, renal stone, human immunodeficiency virus or Hepatitis $B$ virus infections, reflux nephropathy, nephrectomy, solitary kidney, intravenous drug abuse, a family history of renal disease, and patients with FSGS in renal transplant biopsies were also excluded. Renal biopsies containing less than five glomeruli per level of the section in light microscopy were excluded.

\section{Kidney biopsy methods}

Kidney biopsy specimens were evaluated by:

1. Light microscopy on formalin-fixed, paraffin-embedded tissue using hematoxylin and eosin, periodic acid Schiff, and Masson trichrome staining.

2. Immunofluorescence microscopy on frozen tissue using fluoresceinated antibodies to immunoglobulin (lg) G, IgA, IgM, kappa light chains, lambda light chains, C3, C1q, and fibrin.

3. Electron microscopy on tissue fixed in $2.5 \%$ gluteraldehyde and plastic embedded.
Diagnostic criteria:

Criteria to assign each case to a category of the Columbia classification and morphological variants were based exclusively on the paper by D'Agati et al. ${ }^{10}$ The pathologic diagnosis of FSGS was established by the finding of at least one glomerulus with a segmental lesion, and some of the remaining glomeruli were relatively normal. Although the segmental nature of this lesion was usually obvious, we accepted a lesion as segmental when at least one unscarred lobule with patent capillaries was present in the involved glomerulus. ${ }^{11}$ Glomeruli with global sclerosis and glomeruli with segmental lesions were quantified as a percentage of total glomeruli or percentage of viable glomeruli, respectively. The percentage of interstitial fibrosis was semiquantitatively calculated as no fibrosis, mild, moderate or severe, according to Banff schema classification. ${ }^{25}$

\section{Clinical data and definitions criteria:}

Demographic, clinical, and laboratory information at the time of renal biopsy was obtained from each patient. Clinical records were reviewed to determine the Patient's data including gender, age, blood pressure, twenty four hour collection of urine for protein excretion, ${ }^{26}$ serum albumin, blood urea, serum creatinine, ${ }^{27}$ and serum lipid profile ${ }^{28}$ at the time of biopsy. The presentation was defined as the time when proteinuria was first detected. The level of proteinuria at the time of biopsy was categorized as nephrotic-range proteinuria defined as $>3.5$ $\mathrm{g} / \mathrm{d}$ protein and massive proteinuria defined as $>10 \mathrm{~g} / \mathrm{d}$. Nephrotic syndrome was considered for each patient with nephrotic range proteinuria with hypoalbuminemia (serum albumin $<3.5 \mathrm{~g} / \mathrm{dl}$ ). ${ }^{26}$ Hypertension was defined as systolic blood pressure equal to or greater than $140 \mathrm{mmHg}$ and/or diastolic blood pressure equal to or greater than $90 \mathrm{mmHg}$, according to the Seventh Report of the Joint National Committee on Prevention, Detection, Evaluation, and Treatment of High Blood 
Pressure (JNC 7). ${ }^{29}$ Renal insufficiency was defined as a sustained doubling of the serum creatinine, or by initiation of chronic dialysis or kidney transplantation. ${ }^{30}$ The grades of severity of interstitial fibrosis and tubular atrophy (IFTA) is shown according to Banff schema classification. ${ }^{25}$

\section{Statistical analysis}

Data analyzed using the statistical package for social science (SPSS version 19). Fisher s Exact Test was used instead of Chi square test because the expected count of more than $20 \%$ of the cells of tables were less than five. Analysis of variants (ANOVA) was used to compare between three means. A $P$ value of $\leq 0.05$ was considered statistically significant.

\section{Results}

A total of fifty patients are enrolled into this study. The median age of the patients was 33 years, ranged from 18 to 54 (Table 1); $31(62 \%)$ males and 19 (38\%) females. The frequency of histopathological variants was $80 \%$ NOS FSGS, $14 \%$ Glomerular tip lesion FSGS, and $6 \%$ cellular FSGS. There were no identified cases of collapsing and perihilar variants (Table 2). Nephrotic syndrome and hypertension were the main presenting features 46 $(92 \%)$ and $34 \quad(68 \%)$ respectively (Figure 1); severe hypertension constituted $50 \%$ of all hypertensive patients.

Table 1: Distribution of sample by age groups (N-50).

\begin{tabular}{lcc}
\hline Age group & Frequency & Percentage \\
\hline$<25$ & 11 & 22 \\
$25-29$ & 12 & 24 \\
$30-34$ & 3 & 6 \\
$35-39$ & 8 & 16 \\
$40-44$ & 9 & 18 \\
$45+$ & 7 & 14 \\
Total & 50 & 100 \\
\hline
\end{tabular}

Table 2: Frequency distribution of histopathological variants.

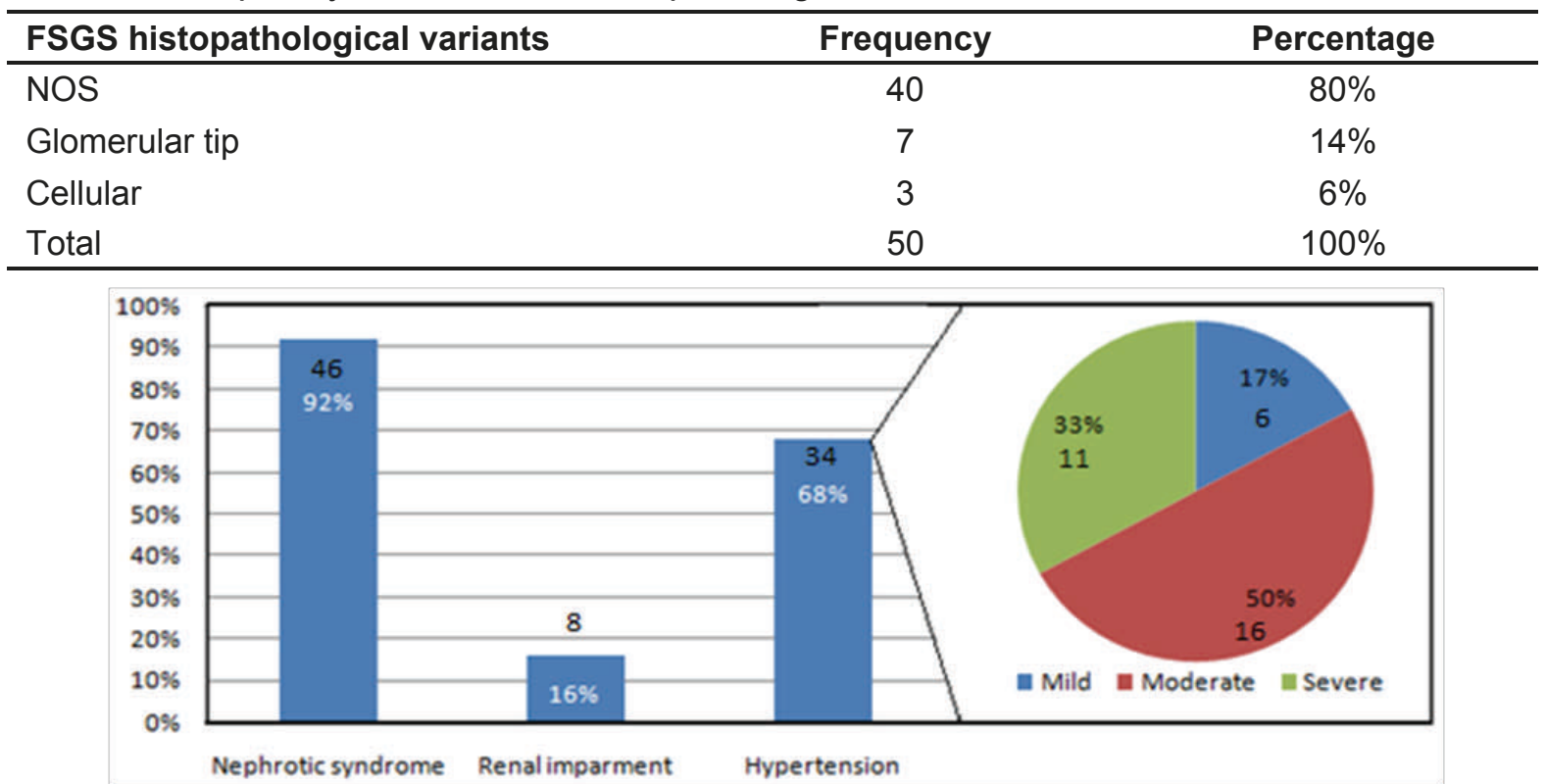

Figure 1: Distribution of sample by clinical characteristics of primary FSGS and grades of hypertension. 
Regarding pathologic findings, the grades of severity of interstitial fibrosis and tubular atrophy (IFTA) are shown in Table 3; the most frequent severity was moderate $20(40 \%)$. The mean percentage of sclerosed glomeruli was $40 \%$ and the mean IFTA\% was $24 \%$. Table 4 shows the sociodemographic, clinical and laboratory characteristics of the three main histopathological variants of FSGS as explained in details separately in the following:

\section{Not otherwise specific FSGS}

In this study, this type was the dominant diagnosed variant among the proved renal biopsy specimen with FSGS. The median age of patients in this group was 33 years (ranged from 18 to 54 ); $60 \%$ were males and $40 \%$ were females ( $\mathrm{M}: \mathrm{F}$ ratio $=3: 2$ ), the mean twenty-four hour urine protein excretion was $(6.95 \mathrm{~g} / \mathrm{d})$, and the mean serum albumin was $(2.9 \mathrm{~g} / \mathrm{dl})$.

\section{Glomerular tip lesion FSGS}

The median age and sex predilection of patients diagnosed with variant were approximately similar to those of NOS variant. Clinically patients are presented with severe nephrotic syndrome with the mean twenty-four hour urine protein excretion of $(9.2 \mathrm{~g} / \mathrm{d})$ which is statistically significant from those with NOS variant $(P=0.027)$, the mean serum albumin was $(2.5 \mathrm{~g} / \mathrm{dl})$ and the mean serum cholesterol level was $268 \mathrm{mg} / \mathrm{dl}$. Renal function tests were least impaired in comparison to other variants patients with a mean serum creatinine of $(0.8 \mathrm{gm} / \mathrm{dl})$ as shown in Table 4.

\section{Cellular FSGS}

All cases are male, mean BP I 186/108, least mean triglyceride $189 \mathrm{mg} / \mathrm{dl}$ and mean cholesterol $212 \mathrm{mg} / \mathrm{dl}$ (Table 4).

Table 3: Frequency distribution of grades of IFTA severity.

\begin{tabular}{lcc}
\hline Grades & Frequency & Percentage \\
\hline Minimum (s5) & 15 & $30 \%$ \\
Mild (6-24) & 13 & $26 \%$ \\
Moderate (25-50) & 20 & $40 \%$ \\
Severe (>50) & 2 & $4 \%$ \\
Total & 50 & $100 \%$ \\
\hline
\end{tabular}

Table 4: Baseline sociodemographic, clinical and laboratory characteristics across the variants

\begin{tabular}{lcccc}
\hline Characteristics & $\begin{array}{c}\text { NOS variant } \\
\mathbf{N = 4 0}\end{array}$ & $\begin{array}{c}\text { Tip variant } \\
\mathbf{N = \mathbf { 7 }}\end{array}$ & $\begin{array}{c}\text { Cellular variant } \\
\mathbf{N = 3}\end{array}$ & $\boldsymbol{P}$ value \\
\hline Age (years) & 33 & 35 & 28 & 0.43 \\
Male & $60 \%$ & $\% 57$ & $100 \%$ & 0.723 \\
Female & $40 \%$ & $43 \%$ & $\% 0$ & \\
Mean BP & $151 / 95$ & $153 / 93$ & $186 / 108$ & 0.727 \\
S.creatinine (mg/dl) & 1.15 & 0.8 & 1.16 & 0.313 \\
Proteinuria (g/d) & 6.5 & 9.2 & 7.6 & 0.027 \\
Massive proteinuria (g/d) & $15 \%$ & $42 \%$ & $0 \%$ & 0.033 \\
S.albumine (g/dl) & 3.2 & 2.5 & 2.7 & 0.037 \\
Cholesterol (mg/dl) & 250 & 268 & 212 & 0.26 \\
Triglyceride (mg/dl) & 273 & 296 & 189 & 0.1 \\
HDL (mg/dl) & 33 & 37 & 42 & 0.153 \\
LDL (mg/dl) & 151 & 184 & 113 & 0.234 \\
\hline
\end{tabular}

$\mathrm{N}=$ number, $\mathrm{HDL}=$ high density lipoprotein, $\mathrm{LDL}=$ low density lipoprotein. 
Regarding the renal function, the pathologic evaluation revealed the least tubulointerstitial injury (the mean IFTA $=2.85 \%)$ which was statistically significant $(P=0.011)$, (Table 5).

\section{Discussion}

Recent studies on clinical-pathologic correlation of patients with FSGS, utilizing the same working protocol of the morphologic classification of FSGS. ${ }^{31-33}$ The study by Stokes et al., 2004 analyzed 47 patients with tip lesion FSGS showed that patients were predominantly Caucasians and often had nephrotic syndrome and severe proteinuria at presentation, similar to patients in our study. In addition, patients often had mild histologic features and preserved renal function. ${ }^{31}$ The study by Chun et al., 2004 compared three FSGS variants, but only two of the variants were defined using the Columbia working proposal criteria. Similar to our results, these patients with tip lesion FSGS more often had nephrotic syndrome and low serum albumin at presentation than those with NOS FSGS. However, the degree of interstitial fibrosis and glomerulosclerosis in their study was not different among their three groups of patients. ${ }^{32}$ The study by Arias et al. studied the presentation and outcome of the different histologic variants of FSGS in Colombia (Hispanic population), revealed that among 291 cases, $77 \%$ corresponded to NOS variant and $13.7 \%$ to tip variant, with a median age of 26 years, pathological findings revealed few histologic chronic lesions in tip lesion cases, and they also found chronic kidney disease was less frequent in tip variant than in the other variants. ${ }^{33}$ The percentage frequency for NOS FSGS $(80 \%)$ was relatively high in this study if we compare it with other series like Chun et al. ${ }^{32}$ We do not know the exact reason; the results may be attributed to the population characteristics or environmental factors. It is very interesting to say that all the other variants may evolve into this pattern in the course of disease progression and increasing chronicity: i.e. it is an unspecific glomerulopathy with many known and unknown etiologies. ${ }^{11}$ According to the definition of cellular variant, the diagnosis requires the presence of endocapillary hypercellularity with occlusion of capillary lumina, with foam cells and/or macrophages and/or endothelial cells and/or neutrophils and/or lymphocytes. ${ }^{10}$ As we can deduce from this microscopic features, in our three cases, lesions were similar to endocapillary proliferative glomerulonephritis (in immune mediated glomerular diseases). It was histological essential in these cases a rigorous examination of immunofluorescence, and electron microscopy, in addition to a thorough review of the clinical manifestation. So, we do not know if the low frequency of this diagnosis is actually due to the rarity of this variant or if the difficulty in its diagnosis contributes to this low frequency. The current study does not define the relative frequency of various FSGS categories exactly because of sampling techniques used that caused a selection bias probably against patients with collapsing and perihilar lesions. The collapsing variant more often presented with a severe nephrotic syndrome and renal impairment. ${ }^{24}$ Therefore, they are more likely to be predicted clinically and sometimes even treated empirically. Regarding the perihilar variant; it is believed that it is commonly associated with secondary forms of hyperfiltration or nephron loss or glomerular hypertension. ${ }^{10}$ This may explain the lack of this variant as

Table 5: Comparison of mean IFTA\% by the variants.

\begin{tabular}{lcc}
\hline Variant & Mean IFTA $\%$ & $\boldsymbol{P}$ value \\
\hline Glomerular tip & $2.86 \pm 7.56$ & \\
Cellular & $33.33 \pm 11.55$ & 0.011 \\
NOS & $24.75 \pm 19.38$ & \\
\hline
\end{tabular}


far as most of these were an exclusion criteria for our study. Our data did not resolve the argument has been raised that the various structural appearances observed in FSGS are merely random patterns of injury that do not define distinct subsets of patients with different disease processes, owing to the lack of significant differences in demographics and clinical presentation that correlate with the different patterns of injury, but this could be attributed to the small sample of cases enrolled in this work as far as all the data were collected prospectively from the time of renal biopsy within a limited period of time. However, the point that supports the distinctiveness of the pathologic variants and for the possibility that they result from different etiologies and pathogenic mechanisms is provided by the correlation of these variants with specific forms of secondary FSGS. For example, known secondary causes of FSGS including human immunodeficiency virus-associated FSGS $^{34}$ and most FSGS caused by pamidronate manifest as collapsing FSGS and not as tip lesion FSGS or perihilar FSGS. $^{13}$ Likewise, perihilar FSGS is the typical glomerular injury caused by obesity $^{35}$ or reduced renal mass. ${ }^{36-37}$ Thus, recognized causes of FSGS usually produce specific patterns of injury that correspond to histologic variants in patients with idiopathic FSGS. This supports the concept that these variants are not random variations caused by a common pathogenic mechanism, but rather are the result of different etiologies.

\section{Conclusion}

The different histologic variants of FSGS have some differences in clinical features at the time of biopsy diagnosis, in spite of that glomerular histological appearance does not permit us to know the cause of FSGS. A not otherwise specific variant is a common morphological lesion in many glomerular and non-glomerular diseases, and it is just like a junk drawer of multiple glomerular alterations with this common pattern of the lesion. The recognition of distinctive variants of disease that are subsumed under the indefinite term FSGS is only a first step toward recognizing etiologically and pathogenetically distinct variants of FSGS, so that appropriate prevention and treatment can be identified.

\section{Conflicts of interest}

The authors report no conflicts of interest.

\section{References}

1. Rich A. A hitherto undescribed vulnerability of the juxtamedullary glomeruli in lipoid nephrosis. Bull Johns Hopkins Hosp 1957; 100:173-86.

2. Churg J, Habib R, White RH. Pathology of the nephrotic syndrome in children: a report for the International Study of Kidney Disease in Children. Lancet 1970; 760:1299-302.

3. Agarwal SK, Dash SC, Tiwari SC, Bhuyan UN. "Idiopathic adult focal segmental glomerulosclerosis: a clinicopathological study and response to steroid." Nephron 1993; 63 (2):168-71.

4. Ponticelli C, Villa M, Banfi G, Cesana B, Pozzi C, Pani $A$, et al. Can prolonged treatment improve the prognosis in adults with focal segmental glomerulosclerosis? Am J Kidney Dis 1999; 34:618-25.

5. Rydel JJ, Korbet SM, Borok RZ, Schwartz MM. Focal segmental glomerular sclerosis in adults: presentation, course and response to treatment. Am J Kidney Dis 1995; 25:534-42.

6. Thomas DB. Focal segmental glomerulosclerosis: a morphologic diagnosis in evolution. Arch Pathol Lab Med 2009; 133(2):217-23.

7. Kitiyakara C, Kopp JB, Eggers P. Trends in the epidemiology of focal segmental glomerulosclerosis. Semin Nephrol 2003; 23:172-82.

8. United States Renal Data System. USRDS 2010 annual data report: atlas of chronic kidney disease and end-stage renal disease in the United States. Bethesda, MD: National Institutes of Health, National Institute of Diabetes and Digestive and Kidney Diseases; 2010.

9. Freedman $\mathrm{BI}$, Hicks $\mathrm{PJ}$, Bostrom MA, Cunningham ME, Liu $Y$, Divers $J$, et al. Polymorphisms in the non-muscle myosin heavy chain 9 gene (MYH9) are strongly associated with end-stage renal disease historically attributed to hypertension in African Americans. Kidney Int 2009; 75(7):736-45.

10. D'Agati VD, Fogo AB, Bruijn JA, Jennette JC. Pathologic classification of focal segmental glomerulosclerosis: a working proposal. Am J Kidney Dis 2004; 43:368-82.

11. Ponticelli C, Glassock RJ. Treatment of Primary Glomerulonephritis. 2nd Ed.UK: Oxford University Press; 2009. p. 215-65. 
12. Friedman EA, Tao TK. Disappearance of uremia due to heroin-associated nephropathy. Am J Kidney Dis 1995; 25(5):689-93.

13. Markowitz GS, Appel GB, Fine PL. Collapsing focal segmental glomerulosclerosis following treatment with high-dose pamidronate. J Am Soc Nephrol 2001; 12:1164-72.

14. Pascual J, Torrealba J, Myers J. Collapsing focal segmental glomerulosclerosis in a liver transplant recipient on alendronate. Osteoporos Int 2007; 1435:8-18.

15. Markowitz GS, Nasr SH, Stokes MB, D'Agati VD. Treatment with IFN $-\alpha,-\beta$, or $-\gamma$ is associated with collapsing focal segmental glomerulosclerosis. Clin J Am Soc Nephrol 2010; 5:607-15.

16. Bruggeman LA, Dikman S, Meng C, Quaggin SE, Coffman TM, Klotman PE. Nephropathy in human immunodeficiency virus-1 transgenic mice is due to renal transgene expression. J Clin Invest 1997; 100(1):84-92.

17. Kimmel PL, Bosch JP, Vassalotti JA. Treatment of human immunodeficiency virus (HIV) -associated nephropathy. Semin Nephrol 1998; 18 (4):446-58.

18. Kumar V, Fausto N, Abbas A. Robbins \& Cotran Pathologic Basis of Disease. $7^{\text {th }}$ ed. Saunders; 2003. p. 982-3.

19. Asanuma K, Mundel P. The role of podocytes in glomerular pathobiology. Clin Exp Nephrol 2003; 7 (4):255-9.

20. Barisoni L, Kriz W, Mundel P, D'Agati V. The dysregulated podocyte phenotype: a novel concept in the pathogenesis of collapsing idiopathic focal segmental glomerulosclerosis and HIV-associated nephropathy. J Am Soc Nephrol 1999; 10(1):51-61.

21. Kriz W. The pathogenesis of "classic" focal segmental glomerulosclerosis-lessons from rat models. Nephrol Dial Transplant 2003; 18:vi39_ 44.

22. Howie AJ, Brewer DB. The glomerular tip lesion: a previously undescribed type of segmental glomerular abnormality. J Pathol 1984; 142:20520.

23. Howie AJ, Brewer DB. Further studies on the glomerular tip lesion: early and late stages and life table analysis. J Pathol 1985; 147:245-55.

24. Detwiler RK, Falk RJ, Hogan SL. Collapsing glomerulopathy: a clinically and pathologically distinct variant of focal segmental glomerulosclerosis. Kidney Int 1994; 45:1416-24.

25. Racusen LC, Solez K, Colvin RB, Bonsib SM, Castro MC, Cavallo T, et al. The Banff 97 working classification of renal allograft pathology. Kidney Int 1999; 55:713-23.

26. Post TW, Rose BD. Urinalysis in the diagnosis of renal disease. In: Curhan GC and Forman JP (Eds). Up To Date 13(2006):3.

27. Lab Tests Online. (Accessed February 10, 2012, at: http://www.labtestsonline.org/understanding/ analytes/urinalysis/tab/sample).
28. National Cholesterol Education Program (Ncep) Expert Panel On Detection, E. "Third Report of the National Cholesterol Education Program (NCEP) Expert Panel on Detection, Evaluation, and Treatment of High Blood Cholesterol in Adults (Adult Treatment Panel III) final report". Circulation 2002; 106(25):3143-421.

29. Chobanian AV. Joint National Committee on Prevention, Detection, Evaluation, and Treatment of High Blood Pressure. National Heart, Lung and Blood Institute; National High Blood Pressure Education Program Coordinating Committee. Seventh report of the Joint National Committee on Prevention, Detection, Evaluation, and Treatment of High Blood Pressure. Hypertension 2003; 42:1206-52.

30. Hsu CY, Chertow GM, Curhan GC. Methodological issues in studying the epidemiology of mild to moderate chronic renal insufficiency. Kidney Int 2002; 61:1567-76.

31. Stokes MB, Markowitz GS, Lin J, Valeri AM, D'agati VD.Glomerular tip lesion: a distinct entity within the minimal change disease/focal segmental glomerulosclerosis sectrum. Kidney Int 2004; 65:1690-702.

32. Chun MJ, Korbet SM, Schwartz MM, Korbert SM and Lewis EJ. Focal segmental glomerulosclerosis in nephrotic adults: presentation, prognosis, and response to therapy of the histologic variants. J Am Soc Nephrol 2004; 15:2169-77.

33. Arias LF, Jiminez CA, Arroyave MJ. Histologic variants of primary focal segmental glomerulosclerosis: presentation and outcome. J Bras Nefrol 2013; 35(2):112-9.

34. Kopp JB, Winkler C. HIV-associated nephropathy in African Americans. Kidney Int Suppl 2003; 63:S43-9.

35. Weisinger JR, Kempson RL, Eldridge FL, Swenson RS. The nephrotic syndrome: a complication of massive obesity. Ann Intern Med 1974; 81:440-7.

36. Gutierrez-Millet V, Nieto J, Praga M, Usera G, Martinez MA, Morales JM. Focal glomerulosclerosis and proteinuria in patients with solitary kidneys. Arch Intern Med 1986; 146:705-9.

37. Praga M, Morales E, Herrero JC. Absence of hypoalbuminemia despite massive proteinuria in focal segmental glomerulosclerosis secondary to hyperfiltration. Am J Kidney Dis 1999; 33:52-8. 